\title{
Ab-initio modelling and docking evaluation of geographically derived coat proteins of chilli leaf curl virus with flavonoids and chemical compounds
}

\author{
Gnanaprakash Jeyaraj, Habeeb Shaik Mohideen, A. Swapna Geetanjali* \\ Department of Genetic Engineering, SRM Institute of Science and Technology, Chennai, India.
}

\section{ARTICLE INFO \\ Article history: \\ Received on: February 05, 2021 \\ Accepted on: April 14, 2021 \\ Available online: September 20,2021}

Key words:

Coat protein AV1, flavonoids, molecular docking, plant virus, chilli leaf curl virus

\section{ABSTRACT}

Chilli leaf curl virus (ChiLCV) belongs to the genus begomovirus (Family Geminiviridae), can infect chilli and many other crops. The coat protein (CP) of ChiLCV binds with the genomic viral ssDNA and shuttles it in and out of the cell nucleus. The distinct role of CP genes is viral capsid formation and transmission by whitefly. In this study, the interaction of $\mathrm{CP}$ with selected plant flavonoids and chemical compounds is explained. The CP of ChiLCV were selected from India, Pakistan, Oman, and Sri Lanka. This study focuses on understanding the structure of CPs from different regions and molecular docking studies to inhibit ssDNA binding for transportation of CP AV1 of ChiLCV. Molecular docking is carried between proteins and selected flavonoids and chemical compounds. Flavonoids show potent inhibition at the binding site of AV1. The outcomes obtained from this study direct that flavonoids' performance promises as compare with other chemical compounds. This information might be interesting to study plant defense mechanisms based on the plants' unique compounds.
\end{abstract}

\section{INTRODUCTION}

Chilli leaf curl virus (ChiLCV) is a destructive virus among chilli varieties worldwide [1]. This viral infection causes a significant economic crisis in the market [2]. The characteristic symptoms are curly leaves, blistered interveins, and stunted growth. Polyphagotarsonemus latus (Mites), Scirtothrips dorsalis (thrips), and Bemisia tabaci (whitefly) are the active vectors of ChiLCV [3]. Chattopadhyay et al. [4] found the infectivity by a complex consisting of monopartite, ChiLCV, and a DNA-beta satellite. Tomato leaf curl Bangladesh beta satellite acts as the factor of chilli leaf curl disease (ChiLCVD). George et al. [5] identified bipartite and monopartite begomovirus association with alpha and beta satellites in ChiLCVD. The satellite, DNA $\beta$, is often associated with the DNA A genome [4]. The right promoter viral strand transcribed the DNA A that codes for coat protein (CP) and pre-coat protein. In contrast, the left promoter transcribed the replication initiator protein $(\mathrm{C} 1)$, transcriptional activator

\footnotetext{
*Corresponding Author

A. Swapna Geetanjali, Department of Genetic Engineering,

SRM Institute of Science and Technology, Chennai, India.

E-mail: swapnaga@srmist.edu.in
}

protein $(\mathrm{C} 2)$, replication enhancer proteins $(\mathrm{C} 3)$, and $\mathrm{C} 4$ protein complementary strand of the sense strand. The pathogenesis and acquired symptoms are due to the single protein $\beta \mathrm{C} 1$ encoded by satellite DNA $\beta[6]$.

Besides, CP is prominent in the vector specificity and viral capsid formation, and vector-mediated transmission [7]. The extermination of viruses or pathogens in the chilli is still challenging due to the limited resources and approaches. By determining the presence of $\mathrm{CP}$ in the chilli plant, we can assure the infection of ChiLCV in the host plant. CP plays a significant role in the virus's attachment and the accumulation of ssDNA into it. Many viral resistance plant studies used CP as the primary source [8].

Structure associated drug design and virtual molecular library screening are highly dependent on the protein structure prediction. 3D structure development was less and a deficit in the area of protein large databases. The three-dimensional models of unknown proteins can be developed by using homology modeling with template protein configurations. Those modeling aiding us in examining the binding loci and drugs suits those binding sites of protein [9]. We are representing four viral proteins from the different geographical areas for which pathological features are 
well studied. This structural prediction emphasizes the in-depth knowledge of virus infection's molecular mechanism [10].

As per the modeller structures, we have to select the inhibitors to block the specific protein's functionality. The natural compounds are preferred over the chemical compounds. The comparison is necessary, and the in silico approach is a productive way to sort out the numerous inhibitors against the viral factor. Natural compounds such as flavonoids are broadly validated in most viral studies [11]. Flavonoids are well known for their antimicrobial activity, and they are the typical components present in almost all plants. The Solanaceae family constitutes different flavonoids, such as kaemferol, quercetin, catechin, and epicatechin are predominant [12]. Six chemical inhibitors such as isoproterenol, riboflavin, atropine, albendazole, neomycin, and ampicillin were evaluated for the tobacco mosaic virus infection, Solanaceae family [13]. Here, we compared the natural flavonoids and chemical inhibitors to shortlist the best active ligand against ChiLCV infection.

Nowadays, it has become easy to guess the molecule binding orientation of drugs, even it was small, and affinity can be calculated [14]. In this study, we carried out the homology modelling for all four coat proteins of ChiLCV from India, Oman, Pakistan, and Sri Lanka. We performed molecular docking against the chemical compound and natural flavonoids using modelling and docking software. Growing in silico studies benefits in vitro studies, such as drug designing, primer designing, and gene silencing [10].

\section{MATERIALS AND METHODS}

\subsection{Sequence Retrieval and ORF Finding}

Protein sequences for ChiLCV of different geographical areas such as India, Oman, Pakistan, and Sri Lanka were retrieved from the GenBank. Specific CP sequence from the whole genome was identified using an ORF finder, and their physicochemical composition was analyzed using ProtPram software. Clustal Omega was used to compare and find the similarity of the sequences with Multiple sequence alignment.

\subsection{Homology Modeling of the Coat Protein}

$A b$ initio 3D models were prepared through the software Phyre 2. From the obtained models, the best was selected from each protein were energy minimized by the YASARA tool. The predicted models are evaluated by the structural analysis and verification server, Molprobity. The Ramachandran plot validates the developed models through the software Molprobity. The models were selected and could be used for further analysis based on the percentage of favor amino acid and outliers' frequency. Active sites of the validated models were predicted using Discover Visualizer studio.

\subsection{Docking with Chemical Compounds and Flavonoids}

\subsubsection{Compound screening}

A total of 14 ligands, including 6 chemical compounds and 8 flavonoids, were obtained from the previous studies. All six compounds were efficient with the notable characters of oral assimilation and absorption of the intestinal epithelium. ADME (Adsorption, Distribution, Metabolism, and Excretion) properties were calculated using SwissADME.

Molecular docking is a key tool to predict protein-ligand interaction. For docking calculations, the PyRx software package with Autodock Vina was employed (https://pyrx.sourceforge. io/). The ligand molecules' structures were downloaded from PubChem and chebi (http://www.ebi.ac.uk/chebi/) in .sdf format and converted to .pdb format using Discover Visualizer studio, which is recognized by PyRx and Autodock Vina. Both selected files of protein and ligands were fed as macromolecules and ligands to PyRx, respectively. The protein and ligand hydrogen were immediately applied using the PyRx hydrogen $(\mathrm{H})$ repair feature. Consequently, the Discovery Studio Visualizer (D.S.V.) will produce a two-dimensional (2D) ligand and target interaction structure.

\section{RESULTS AND DISCUSSION}

\subsection{Sequence Retrieval and Physiochemical Parameters}

The retrieved nucleotide sequence of $\mathrm{CP}$ was translated into protein sequence through smart blast in the ORF finder were shown in Table 1. In the secondary structure prediction, the different amino acids present in the CP gene of ChiLCV with physiological and chemical parameters. The molecular weight, number of amino acids, and theoretical pI values were listed in Table 2. The CP multiple sequence alignment shows that sequences share high similarity and all the conserved regions are the same (Fig. 1).

\subsection{Ab-initio Modelling of Coat Protein and Validation of the 3D Model}

A 3D model of the ChiLCV CP from Oman, India, Sri Lanka, and Pakistan were created in the present research. The model was developed using the ab initio homology modelling via the Phyre 2 server (Fig. 2). The validation of all four coat proteins performed by the Ramachandran plot was shown in Figure 3. The characteristics of the models were listed in Table 2.

\subsection{Lipinski Rule and ADME Prediction}

Significant properties of ADME like water solubility, level of human oral absorption in GI, Blood-Brain barrier permanent level,

Table 1: Retrieved sequence accession no and Protein ID.

\begin{tabular}{llcc} 
Virus name & Accession no & Protein name & Protein accession no \\
Chilli leaf curl virus isolate CapAS 2, complete genome & KM023148.1 & Coat protein & AIR77200.1 \\
Chilli leaf curl virus clone Wat-122, complete genome & KX787939.2 & Coat protein & APC65287.2 \\
Chilli leaf curl virus complete genome, clone RM270 & LN886652.1 & Coat protein & CUR29891.1 \\
Chilli leaf curl virus isolate CL-15 from Sri Lanka, complete genome & JN555600.1 & Coat protein & AEY77827.1 \\
\hline
\end{tabular}


Table 2: Physiological and chemical analysis of predicted coat protein of ChiLCV from different geographical areas.

\begin{tabular}{lcc} 
Physiological and chemical parameters & Value & Geographical area \\
Number of amino acids & 257 & Oman \\
Molecular weight & $29,714.19$ & \\
Theoretical pI & 10.12 & \\
Total number of negatively charged residues (Asp + Glu) & 21 & India \\
Total number of positively charged residues (Arg + Lys) & 44 & \\
Number of amino acids & 256 & \\
Molecular weight & $29,664.22$ & Sri Lanka \\
Theoretical pI & 10.06 & \\
Total number of negatively charged residues (Asp + Glu) & 21 & \\
Total number of positively charged residues (Arg + Lys) & 44 & \\
Number of amino acids & 256 & \\
Molecular weight & $29,581.26$ & Pakistan \\
Theoretical pI & 9.99 & \\
Total number of negatively charged residues (Asp + Glu) & 21 & \\
Total number of positively charged residues (Arg + Lys) & 44 & 256 \\
Number of amino acids & $29,687.15$ & 10.08 \\
Molecular weight & 22 & \\
Theoretical pI & 44 & \\
Total number of negatively charged residues (Asp + Glu) & & \\
Total number of positively charged residues (Arg + Lys) & & \\
\hline
\end{tabular}

a)

\begin{tabular}{|c|c|c|}
\hline ADO95313.1:1-256 & MSKRPADI IISTPASKVRRL LNFDSPYTSRAAAP IVRVTKAKAWANRPMNRKPRMYRMYR & 60 \\
\hline ALN96432.1:1-256 & & 60 \\
\hline ADQ13194.1:1-256 & & $6 \oplus$ \\
\hline AIA58521.1:1-256 & 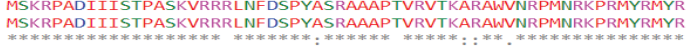 & 60 \\
\hline ADO95313.1:1-256 & SPDVPRGCEGPCKVQSFESRHDTQHIGKVMCVSDATRGTGLTHRVGKRFCVKSVYVLGKI & 120 \\
\hline ALN96432.1:1-256 & \multirow{2}{*}{ 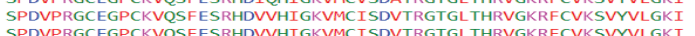 } & \\
\hline ADQ13194.1:1-256 & & 120 \\
\hline AIA58521.1:1-256 & SPDVPRGCEGPCKVQSFESRHDVVHIGKVMCI ISDVTRGTGLTHRVGKRFCVKSVYVLGKI & 120 \\
\hline & & \\
\hline AD095313.1:1-256 & WMDENIKTKNHTNSVMFFLVRDRRPVDKPODFGEVFNMFDNEPSTATVKMMHRDRYOVLR & 180 \\
\hline ALN96432.1:1-256 & WMDENIKT KNHTNS VMF FLVRDRR RP TDKPQDFGEVFNMFDNEPSTATVKNMHRDR YQVLR & 180 \\
\hline ADQ13194.1:1-256 & WMDENIKTKNHTNSVMF FLVRDRRPVDKPQDFFGEVFNMFDNEPSTATVKNMMHRDRYQVLR & 180 \\
\hline AIA58521.1:1-256 & 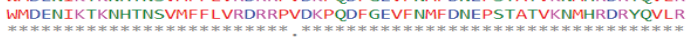 & \\
\hline & & \\
\hline ALN96432.1:1-256 & \multirow{2}{*}{ 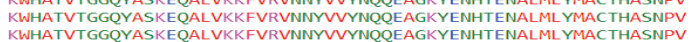 } & 240 \\
\hline ADQ13194.1:1-256 & & 240 \\
\hline ATA58521.1:1-256 & KWHATVTGGQYASKEQALWKKFVRVNNYVYNQQEAGKYENHTENALML YMACTHASNPV & 240 \\
\hline & & \\
\hline $\begin{array}{l}\text { ADO95313.1:1-256 } \\
\text { ALN96432.1:1-256 }\end{array}$ & \\
\hline ADQ13194.1:1-256 & & \\
\hline AIA58521.1:1-256 & \multicolumn{2}{|l|}{$\begin{array}{l}\text { YATLKIRIYFYDSVSN } 256 \\
\text { YATLKTRTYFFDSVSN } 256\end{array}$} \\
\hline
\end{tabular}

\section{b)}
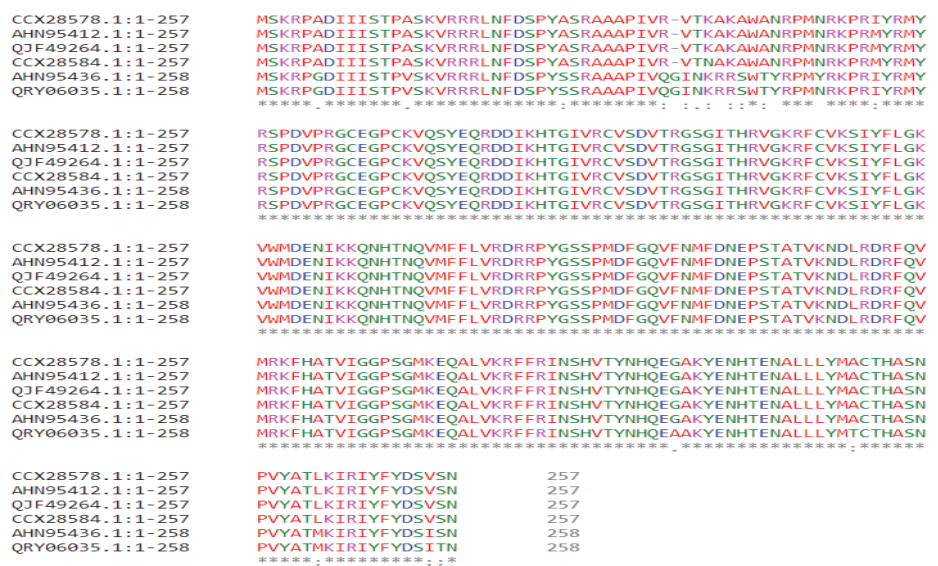

59
59
59
59
60
60 
c)

\begin{tabular}{|c|c|c|c|}
\hline ALQ43532.1:1-256 & \multicolumn{2}{|c|}{ MSKRPTDIIISTPASKVRRRLNFDSPYASRAAVPTVRVIKARAWANRPMNRKPRMYRMYR } & \\
\hline AIC83088.1:1-256 & \multirow{2}{*}{\multicolumn{2}{|c|}{ 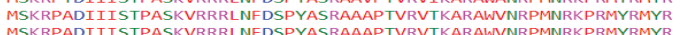 }} & 60 \\
\hline CUR29891.1:1-256 & & & 60 \\
\hline YP_005352657.1:1-256 & \multicolumn{2}{|c|}{ MSKRPADI IISTPASKVRRRLNLDSPYASRAAAPIVRVTKARAWANRPMNRKP RMYRMYR } & 60 \\
\hline САМ̈33.228.1:1-256 & \multicolumn{2}{|c|}{ 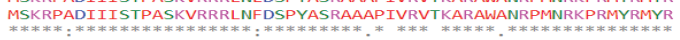 } & 60 \\
\hline ALO43532.1:1-256 & \multicolumn{2}{|c|}{ SPDVPRGCEGPCKVQSFESRHDVVHIGKVMCISDVTRGTGLTHRVGKRFCVKSVYVLGKI } & \\
\hline AIC $83088.1: 1-256$ & \multicolumn{2}{|c|}{ 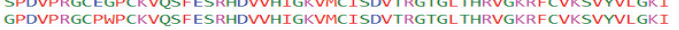 } & 120 \\
\hline CUR29891.1:1-256 & \multirow{2}{*}{\multicolumn{2}{|c|}{ SPDVPRGCEGPCKVRSFESRHDVVHIGKVMCISDVTRGTGLTHRVGKRFCVKSWYVLGKI }} & 120 \\
\hline YP_Q105352657.1:1-256 & & & 120 \\
\hline CAM $33228.1: 1-256$ & \multicolumn{2}{|c|}{ SPDVPRGCEGPCKVQSFESRHDVVHIGKVMCISDVTRGTGLTHRVGKRFRVKSVYVLGKI } & 120 \\
\hline & \multicolumn{2}{|c|}{ WMDENT KTKNHTNSVMFFL VRDRRPVDKPODFGEVFNMFDNEPSTATVKNLHRDRYOVLR } & \\
\hline $\begin{array}{l}\text { LQ443532.1:1-256 } \\
\text { IC83088.1:1-256 }\end{array}$ & \multirow{3}{*}{\multicolumn{2}{|c|}{ 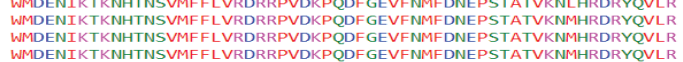 }} & \\
\hline $\begin{array}{l}\text { ALC } 33088.1: 1-256 \\
\text { CUR29891.1:1-256 }\end{array}$ & & & 180 \\
\hline $\begin{array}{l}\text { CUR298891.1:1-256 } \\
\text { YP } 005352657.1: 1-256\end{array}$ & & & 180 \\
\hline САМ $33228.1: 1-256$ & \multicolumn{2}{|c|}{ 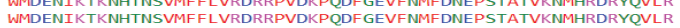 } & \\
\hline ALQ43532.1:1-256 & \multirow{2}{*}{\multicolumn{2}{|c|}{ KWHATVTGGQYASKEQALVKKFVRVNNYVVYNQOEAGKYENHTENALMLYMACTHASNPV }} & \\
\hline AIC83088.1:1-256 & & & 240 \\
\hline CUR29891.1:1-256 & \multicolumn{2}{|c|}{ 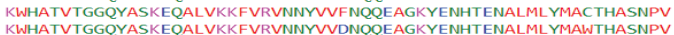 } & 240 \\
\hline YP_005352657.1:1-256 & \multicolumn{2}{|c|}{ KWHATVTGGQYASKEQALVKKF VRVNN YVVDNOQEAGKYENHTENALMLYMAWTHASNPV } & 240 \\
\hline CAM $33228.1: 1-256$ & \multicolumn{2}{|c|}{ 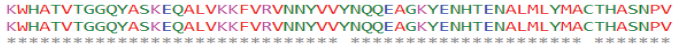 } & \\
\hline & & & \\
\hline AIC83088.1:1-25 & YFYDSVSN & 256 & \\
\hline $\begin{array}{l}\text { CUR29891.1:1-25 } \\
\text { CUR }\end{array}$ & LLKIRIYFYDSVSN & 256 & \\
\hline YP_005352657.1:1-256 & YATLKIRIYFYDSVSN & 256 & \\
\hline CAM33228.1:1-256 & YATLKIRIYFYDSVSN & 256 & \\
\hline
\end{tabular}

d)

CLUSTAL O(1.2.4) multiple sequence alignment

\begin{tabular}{|c|c|}
\hline $\begin{array}{l}\text { MP } 898823.1: 1-256 \\
\text { AEV } 777827.1: 1.256 \\
\text { AEY77833.1:1-256 }\end{array}$ & $\begin{array}{lll} & \text { N }\end{array}$ \\
\hline $\begin{array}{l}\text { NP }-808823.1: 1-256 \\
\text { AEVT7827 } 1: 1: 1256 \\
\text { AEY777833:1:1-256 }\end{array}$ & 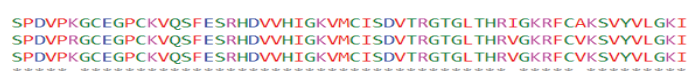 \\
\hline 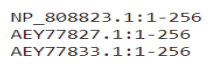 & 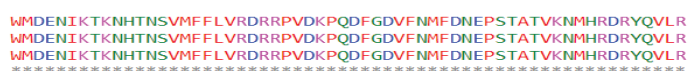 \\
\hline $\begin{array}{l}\text { NP- } 888823.1: 1-256 \\
\text { AEV77827.1:1-256 } \\
\text { AEY77833.1:1-256 }\end{array}$ & 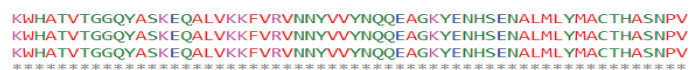 \\
\hline $\begin{array}{l}\text { NP }-808823.1: 1-256 \\
\text { AEV77827.1:1-256 } \\
\text { AEY77783.1:1-256 }\end{array}$ & 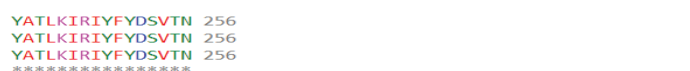 \\
\hline
\end{tabular}

Figure 1: Multiple sequence alignment of retrieved coat protein sequences with other protein sequences a) India b) Oman c) Pakistan d) Sri Lanka.

A
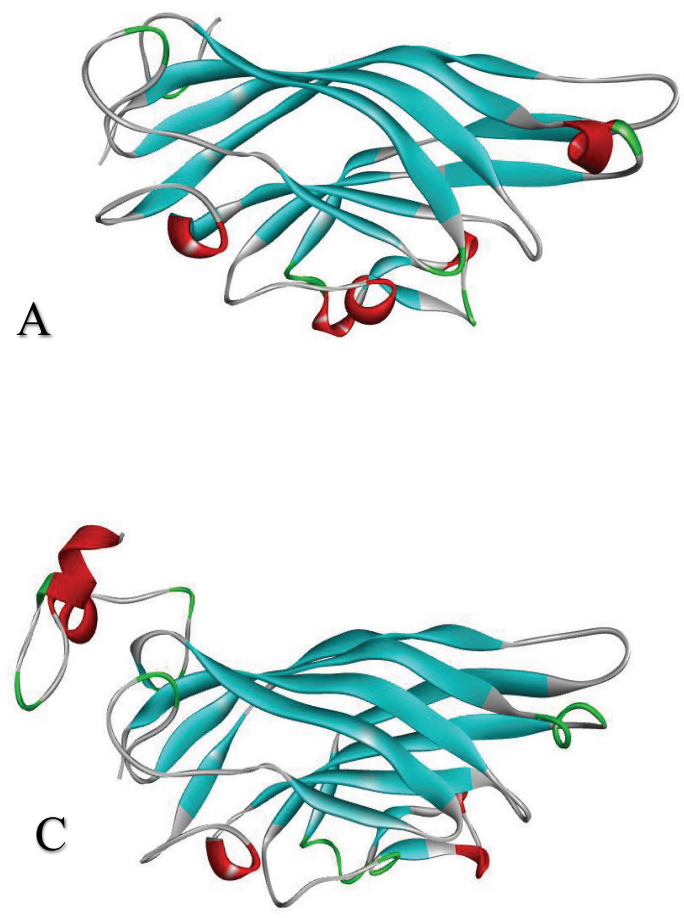

B

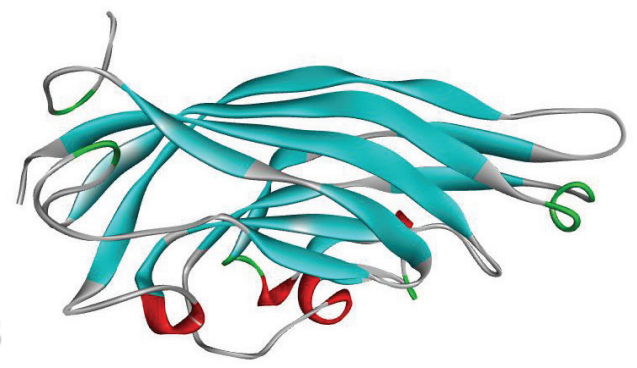

$\mathrm{D}$

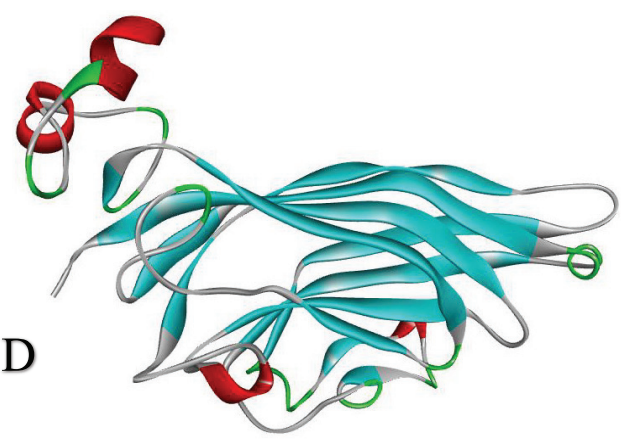

Figure 2: Ab initio modeling of coat proteins. a) India, b) Oman, c) Pakistan, and d) Sri Lanka. 

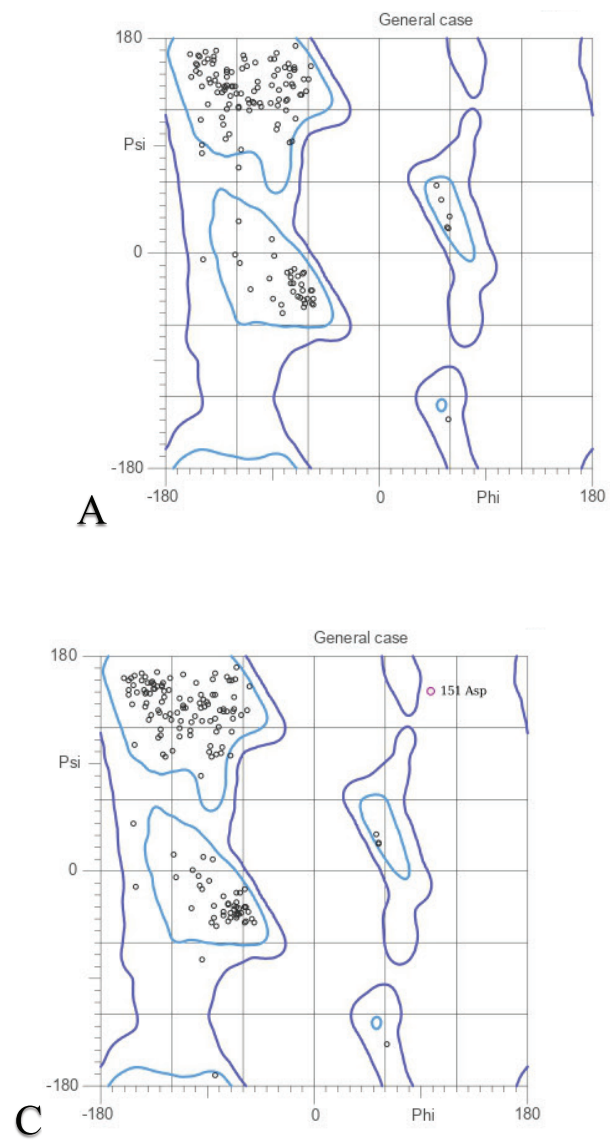
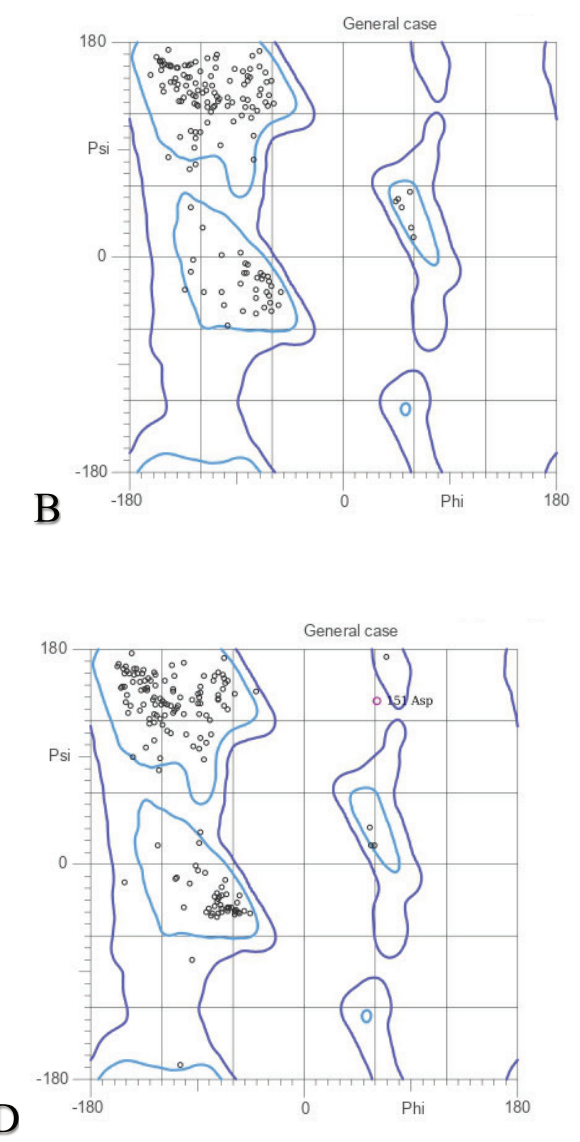

Figure 3: Ramachandran plot obtained using Molprobity. a) India, b) Oman, c) Pakistan, and d) Sri Lanka.

Table 3: Structure validation scores using Molprobity server for the coat protein of ChiLCV.

\begin{tabular}{|c|c|c|c|c|c|}
\hline S. No & Parameters & India & Oman & Pakistan & Sri Lanka \\
\hline 1 & No. of residues in favored region & $186(96.37 \%)$ & $188(96.91 \%)$ & $206(95.81 \%)$ & $186(96.37 \%)$ \\
\hline 2 & No. of residues in outlier region & $0(0 \%)$ & $1(0.52 \%)$ & $3(1.40 \%)$ & $0(0 \%)$ \\
\hline 3 & Ramachandran Z- score & $-0.01 \pm 0.58$ & $-1.29 \pm 0.59$ & $-0.061 \pm 0.51$ & $-0.01 \pm 0.58$ \\
\hline
\end{tabular}

skin permeable level, bioavailability, and Lipinski violations of selected inhibitors were tabulated in Table $4 \mathrm{a}$ and $\mathrm{b}$.

\subsection{Docking Studies}

Discovery Studio Visualizer recognized the active site regions in the modelled proteins. Docking studies of selected chemical and natural compounds were completed using PyRx docking software. The selected vitamins as the chemical compounds for coat protein interaction are well-known activators in different plants' defense mechanisms [15], as well as flavonoids functions against bacterial infection [16]. Flavonoids represent the active player as signaling molecules in the plant microorganism interactions [17]. The characteristic functions of flavonoids are detoxification agents, scavenge ROS, $\mathrm{H}_{2} \mathrm{O}_{2}$, and toxic metals and, radiation stress suppressors $[18,19]$. These flavonoids are borne defense mechanisms and function as phytoalexins to respond against microbial infections [20]. The findings were analyzed, the binding energy and the hydrogen bonding regions were established after the docking studies were performed, as shown in Table 5a and b.

\subsection{Calculations of Ligand Interaction}

Discovery Studio Visualizer calculated interactions between proteins with ligands. The interaction of chemicals and flavonoids with each protein offers a means to examine each atom of ligands and proteins involved in this interaction. As compare with selected chemical compounds, flavonoids showing good affinity with proteins. A portrayed view of $2 \mathrm{D}$ interaction was shown in Figure 6. India_coat protein affinity with ampicillin has two hydrogen bonds and one weak pi-pi interaction, although Agathisflavone forms one ionic bond and pi-pi interaction bond. An interaction with Oman_coat protein Agathisflavone and albendazole in flavonoids were shown promising results. Agathisflavone formed an interaction with three amino acids by two hydrogen bonds and ionic bonds, whereas albendazole has four hydrogen bonds and 
Table 4: a) ADME properties of flavonoid compounds.

\begin{tabular}{|c|c|c|c|c|c|c|c|c|}
\hline Molecule & MW & $\begin{array}{l}\text { ESOL } \\
\log S\end{array}$ & ESOL class & $\begin{array}{c}\text { GI } \\
\text { absorption }\end{array}$ & $\begin{array}{c}\text { BBB } \\
\text { permeant }\end{array}$ & $\begin{array}{l}\log K p \\
(\mathrm{~cm} / \mathrm{s})\end{array}$ & $\begin{array}{c}\text { Lipinski } \\
\text { \#violations }\end{array}$ & $\begin{array}{l}\text { Bioavailability } \\
\text { Score }\end{array}$ \\
\hline Luteolin 7-O-beta-Dglucosiduronic Acid & 462.36 & -3.41 & Soluble & Low & No & -8.43 & 2 & 0.11 \\
\hline Quercetin 7-O-beta-Dglucoside & 464.38 & -3.04 & Soluble & Low & No & -8.88 & 2 & 0.17 \\
\hline Swertianolin & 436.37 & -3.01 & Soluble & Low & No & -8.52 & 2 & 0.17 \\
\hline Prunin 600 -O-gallate & 586.5 & -4.13 & Moderately soluble & Low & No & -8.98 & 3 & 0.17 \\
\hline 3,4,5-trihydroxy-3-methoxyflavon-7-olate & 315.25 & -3.88 & Soluble & High & No & -6.3 & 0 & 0.56 \\
\hline
\end{tabular}

Table 4: b) ADME properties of chemical compounds.

\begin{tabular}{lcccccccc} 
Molecule & MW & ESOL Log $S$ & ESOL Class & GI absorption & BBB permeant & log Kp (cm/s) & $\begin{array}{c}\text { Lipinski } \\
\text { \#violations }\end{array}$ & $\begin{array}{c}\text { Bioavailability } \\
\text { Score }\end{array}$ \\
Albendazole & 376.36 & -1.31 & Very soluble & Low & No & -9.63 & 0 & 0.55 \\
Ampicillin & 349.4 & -1.15 & Very soluble & Low & No & -9.23 & 0 \\
Atropin & 289.37 & -2.67 & Soluble & High & Yes & -6.77 & 0.55 & 0.55 \\
Iso & 614.64 & 2.61 & Highly soluble & Low & No & -16.43 & 3 & 0.17 \\
Neomycin & 211.26 & 1.02 & Highly soluble & High & No & -10.07 & 0 \\
Riboflavin & 265.33 & -3.23 & Soluble & High & No & -5.92 & 0.55 \\
\hline
\end{tabular}<smiles>COc1cc2oc(-c3ccc(O)c(O)c3)c(OC)c(=O)c2c(O)c1O</smiles>

A<smiles>Cc1ccc(-c2cc(=O)c3c(O)c(-c4c(O)cc(O)c5c(=O)cc(-c6ccc(O)cc6)oc45)c(O)cc3o2)cc1</smiles>

B<smiles>O=c1cc(-c2ccc(O)c(O)c2)oc2cc(O[C@@H]3OC(CO)[C@H](O)[C@H](O)C3O)cc(O)c12</smiles><smiles>COc1cc(-c2cc(=O)c3c(O)cc(O)cc3o2)ccc1[O-]</smiles> 


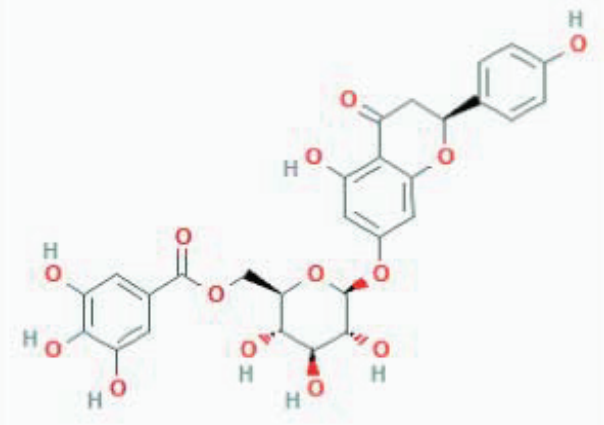

$\mathrm{E}$

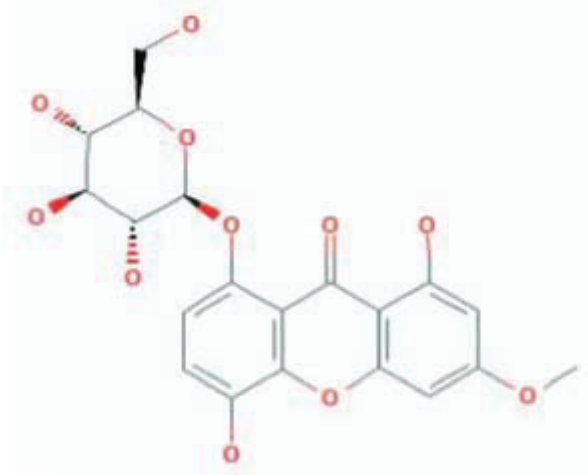

Gi

Figure 4: 2D structure of ligands. A) 30,40,5-trihydroxy-3-methoxyflavon-7-olate, B) Prunin 600-O-gallate, C) Catiguanin B, D) Swertianolin, E) Quercetin 7-O-beta-dglucoside, F) 30,40,5,6-tetrahydroxy-3,7-dimethoxyflavone, G) Luteolin 7-O-betadglucosiduronic acid, and $\mathrm{H}$ ) Agathisflavone. an ionic bond. A CP obtained from the Pakistan sequence has five hydrogens. One ionic bonding with ampicillin and prunin 600-O-gallate has interacted with CP by forming four hydrogen bonds that include 1 weak hydrogen bond, one pi-pi interaction bond, and finally, one unfavorable donor-donor interaction with TYR219. Agathisflavone makes 3 weak pi-pi interaction bonds, three hydrogen bonds, and one ionic bond with Sri Lanka CP. Even albendazole has four hydrogen bonds, one ionic, one Pi-sulfur bond, and unfavorable donor-donor (CYS91) with Sri Lanka CP. The interaction between proteins and ligands with distance values were shown in 2D Figures.

RNA interference (RNAi), systemic antiviral defense, DNA methylation, neurotic resistance, systemic acquired resistance response development, secondary metabolites in plants are the plants' viral immune response against pathogens [21]. Flavonoids showed a significant defense against coat proteins in comparison. The plant develops flavonoids while it indulges in stress conditions [22]. Flavonoids serve as a conventional eastern medicine with anti-inflammatory, antioxidant, anti-tumor, and antiproliferative activities [23].
Our research examined plant flavonoids and chemical inhibitors using the computational-based docking system with pathogenicity determinant CP, AV1. As a need of three-dimensional structure of the protein for docking analysis. There was no determination of the structure of the AV1 protein, so we developed this protein model by homology modelling method.

Different online servers then analyzed the consistency and stereochemistry of the computer-generated model of AV1. For docking analysis, the protein structure in the .pdb format is used. The binding energy and the optimal posture of the molecule for protein binding are expected in the docking study. Our emphasis in the current study was to discover the role of natural flavonoids and chemical inhibitors against the AV1 CP. For this reason, eight flavonoids, i.e., Swertianolin, 3',4',5-trihydroxy-3-methoxyflavon7-olate, Agathisflavone, Catiguanin B, 3',4',5,6-tetrahydroxy-3,7dimethoxyflavone, quercetin- 7-O-[alpha-L-rhamnopyranosyl(1$>6$ )-beta-D-galactopyranoside], prunin 600-O-gallate and luteolin 7-O-beta-D-glucosiduronic acid, were obtained as a potential inhibitor of AV1 based on best docking scores against the active site of AV1. These flavonoids have long been known to suppress 


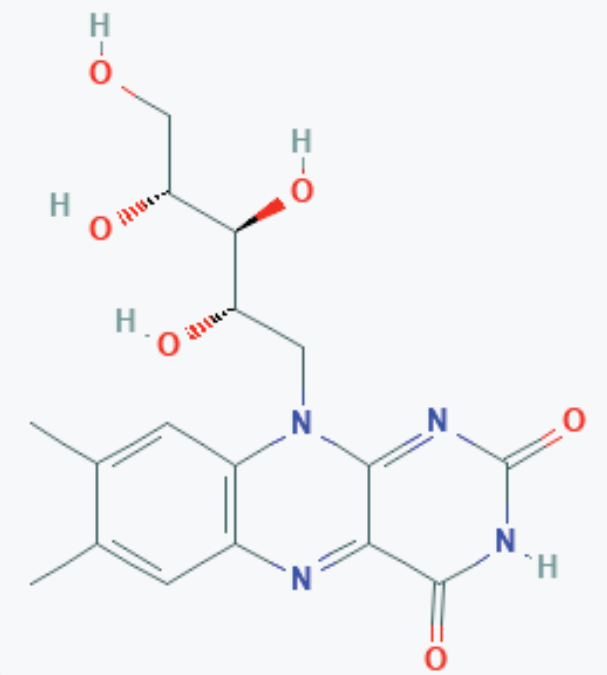

A<smiles>CC(C)NCC(O)c1ccc(O)c(O)c1</smiles><smiles>CC1(C)S[C@@H](NNC(=O)[C@@H](N)c2ccccc2)[C@H](C(=O)O)N1C(=O)O</smiles>

E

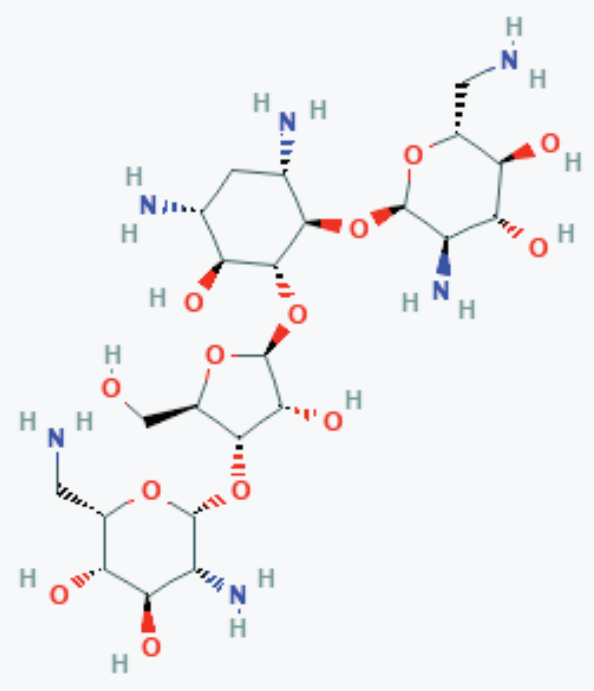

B

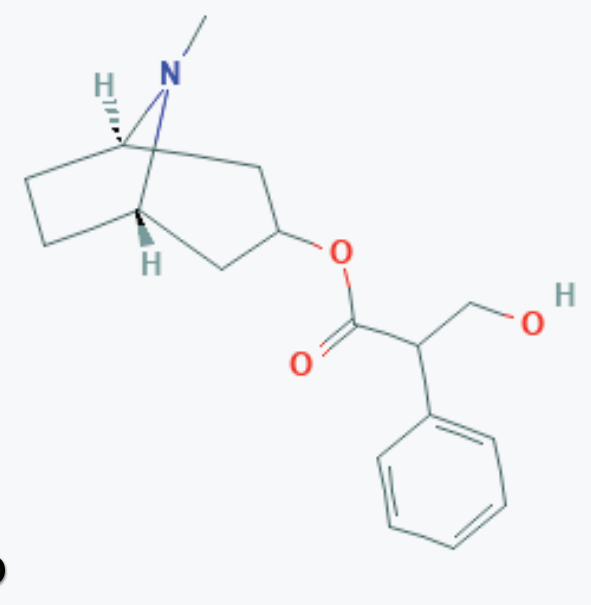


Table 5: a) Binding energy $(\mathrm{kcal} / \mathrm{mol})$ of ligands with proteins (flavonoids).

\begin{tabular}{lcccc} 
Compound Chem ID & $\begin{array}{c}\text { India } \\
\text { (kcal/mol) }\end{array}$ & $\begin{array}{c}\text { Oman } \\
(\mathbf{k c a l} / \mathbf{m o l})\end{array}$ & $\begin{array}{c}\text { Pakistan } \\
\text { (kcal/mol) }\end{array}$ & $\begin{array}{c}\text { Sri Lanka } \\
\text { (kcal/mol) }\end{array}$ \\
\hline Agathisflavone_2512 & -8.2 & -7.6 & -7.3 & -7.0 \\
Luteolin 7-O-beta-dglucosiduronic Acid_18128 & -4.2 & -4.6 & -4.5 & -4.5 \\
3,4,5,6-tetrahydroxy-3,7-dimethoxyflavone_27767 & -6.1 & -6.2 & -6.0 & -6.3 \\
Quercetin 7-O- $\beta$-D-glucoside_28529 & -3.9 & -4.3 & -4.1 & -4.2 \\
$\quad$ Swertianolin_ 65478 & -3.9 & -4.3 & -4.1 & -4.2 \\
Catiguanin B_65602 & -7.3 & -6.3 & -6.9 & -6.5 \\
Prunin 600-O-gallate 73787 & -7.6 & -7.5 & -7.5 & -6.8 \\
30,40,5-trihydroxy-3-methoxyflavon-7-olate57928 & -5.8 & -6.0 & -6.0 & -5.9 \\
\hline
\end{tabular}

Table 5: b) Binding energy $(\mathrm{kcal} / \mathrm{mol})$ of ligands with proteins. (Chemical compounds).

\begin{tabular}{lcccc} 
Compound & India $(\mathbf{k c a l} / \mathbf{m o l})$ & Oman $(\mathbf{k c a l} / \mathbf{m o l})$ & Pakistan $(\mathbf{k c a l} / \mathbf{m o l})$ & Sri Lanka $(\mathbf{k c a l} / \mathbf{m o l})$ \\
Albendazole & -3.9 & -5.3 & -6.0 & -6.1 \\
Ampicillin & -4.6 & -5.2 & -6.1 & -5.9 \\
Atropine & -4.0 & -4.6 & -5.6 & -5.4 \\
Isoproterenol & -0.2 & 1.3 & -5.5 & -5.5 \\
Neomycin & -3.9 & -5.0 & -4.8 & -4.9 \\
Riboflavin & -3.9 & -4.7 & -5.0 & -5.0 \\
\hline
\end{tabular}
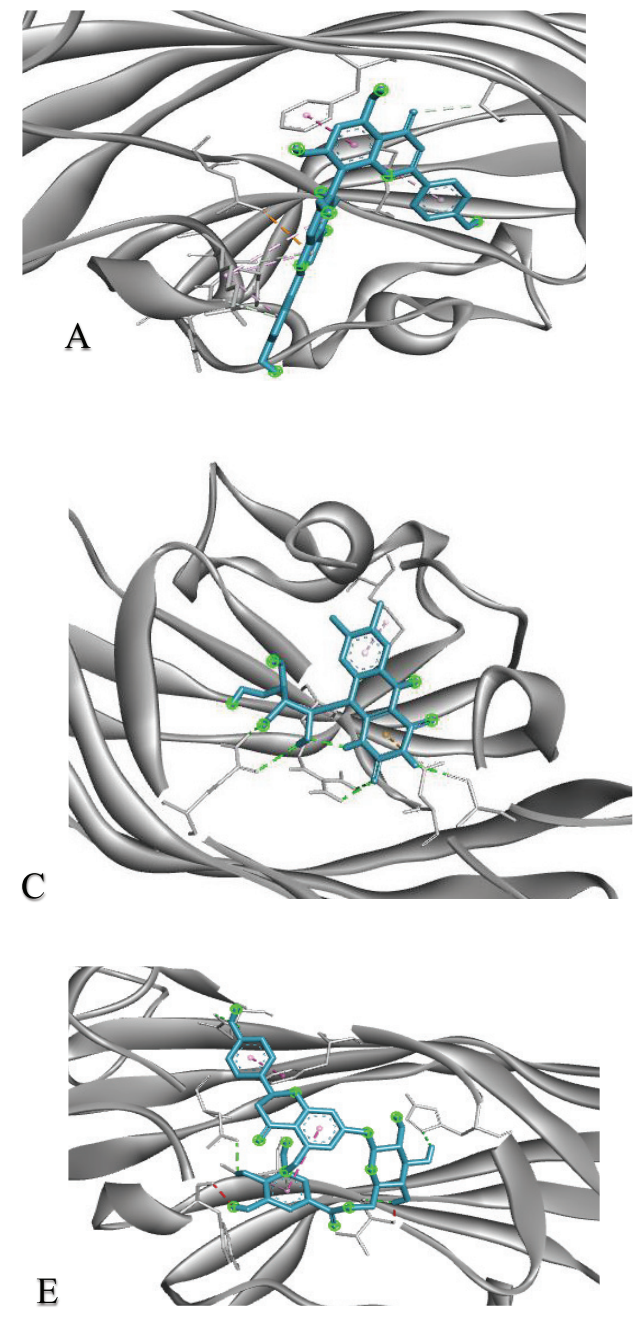

B

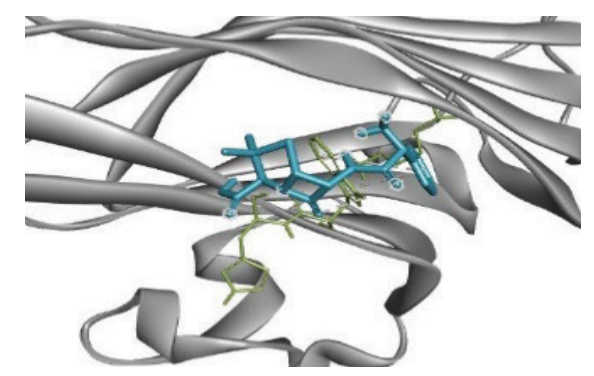

D

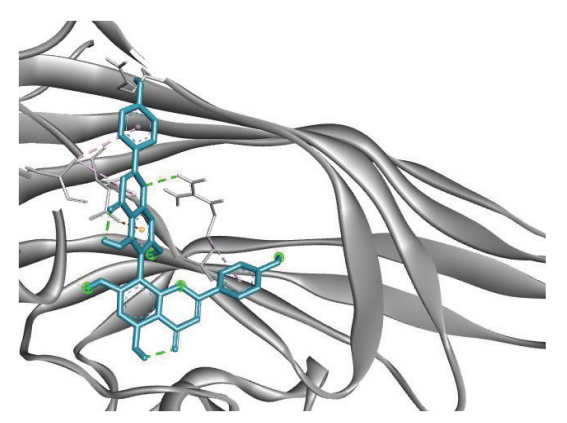

$\mathrm{F}$

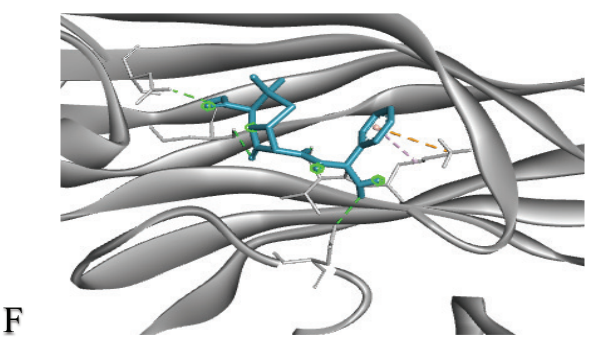



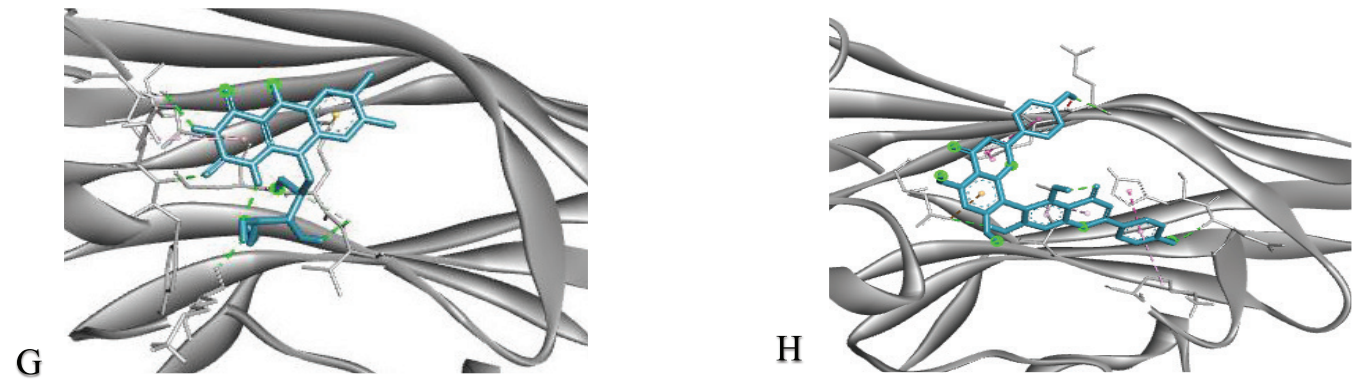

Figure 6: 3D structure of proteins interacting with Ligands. A) and B) India_coat protein with Agathisflavone and Ampicillin. C) and D) Oman_coat protein with ligand Agathisflavone and Albendazole. E) and F) Pakistan_coat protein with ligand Prunin 600-O-gallate and ampicillin. G) and H) Srilanka_coat protein with ligand Agathisflavone and Ampicillin.
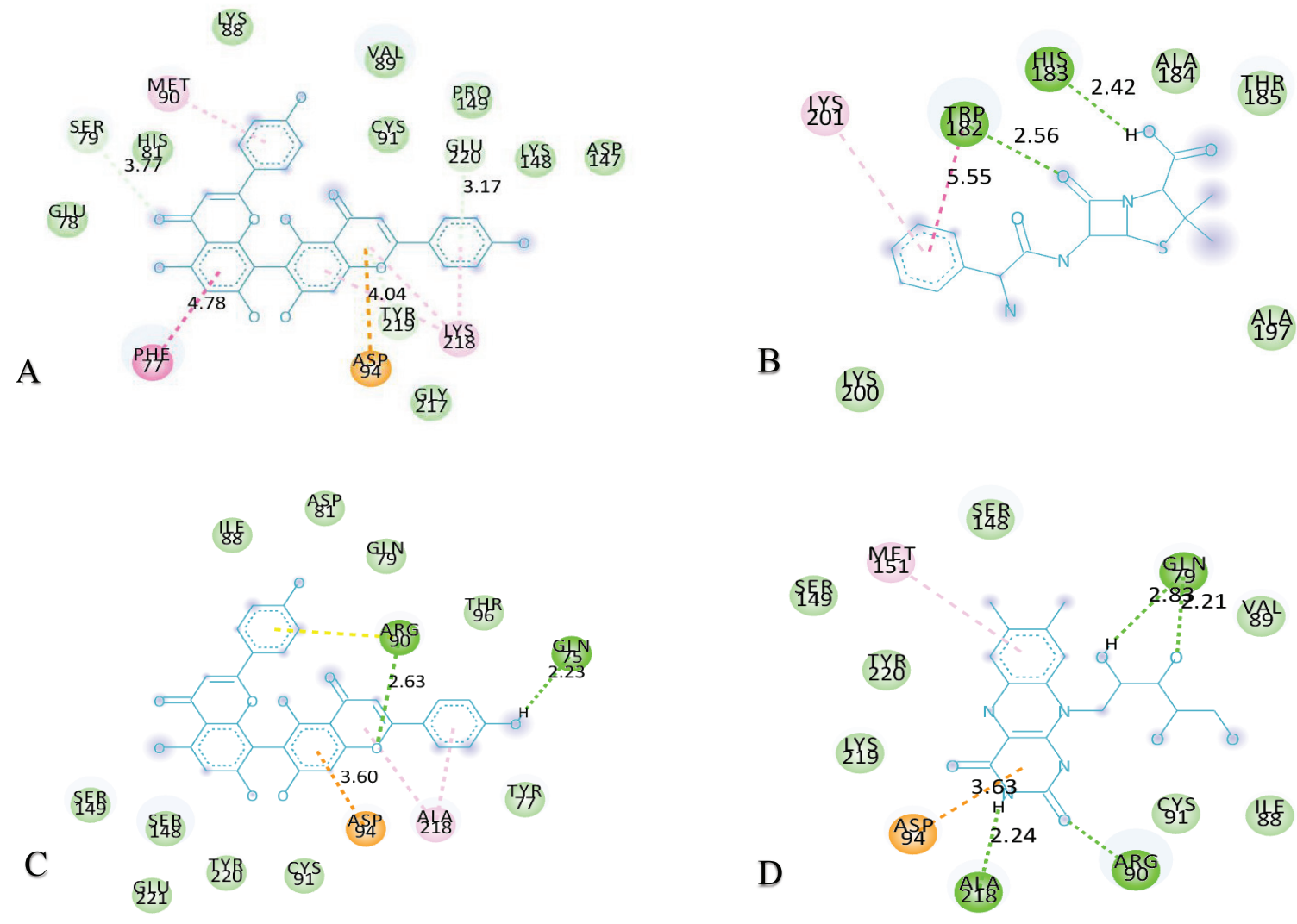

B 205

A19A

ASP
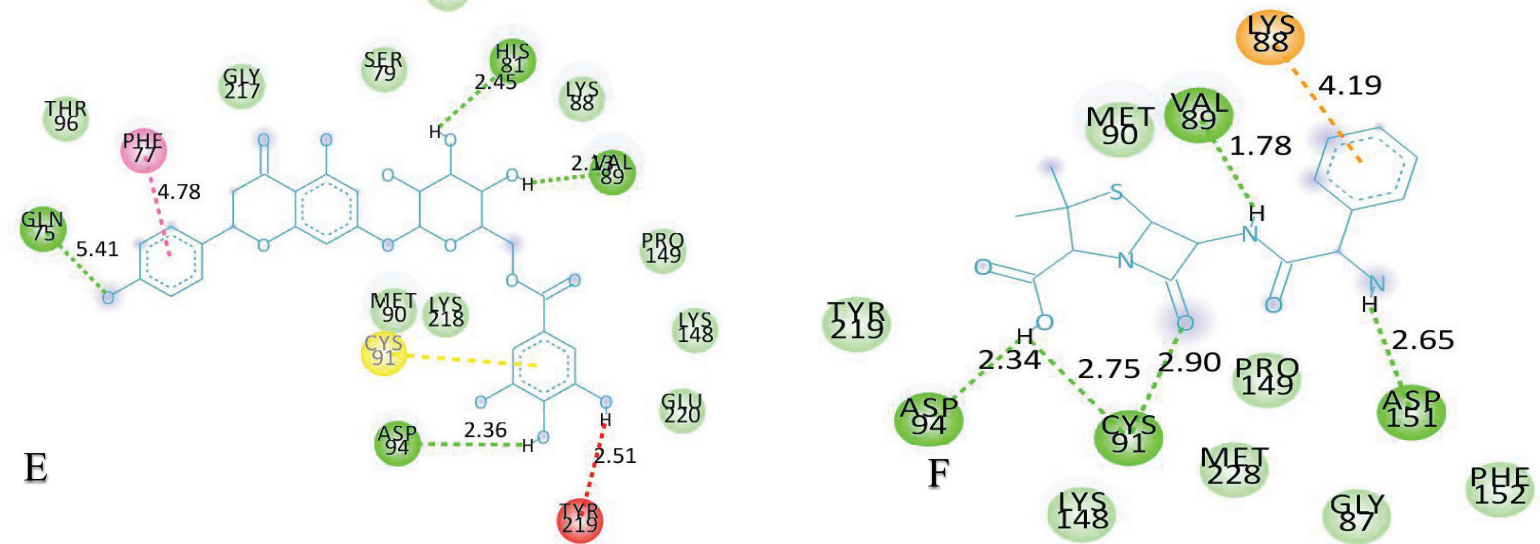

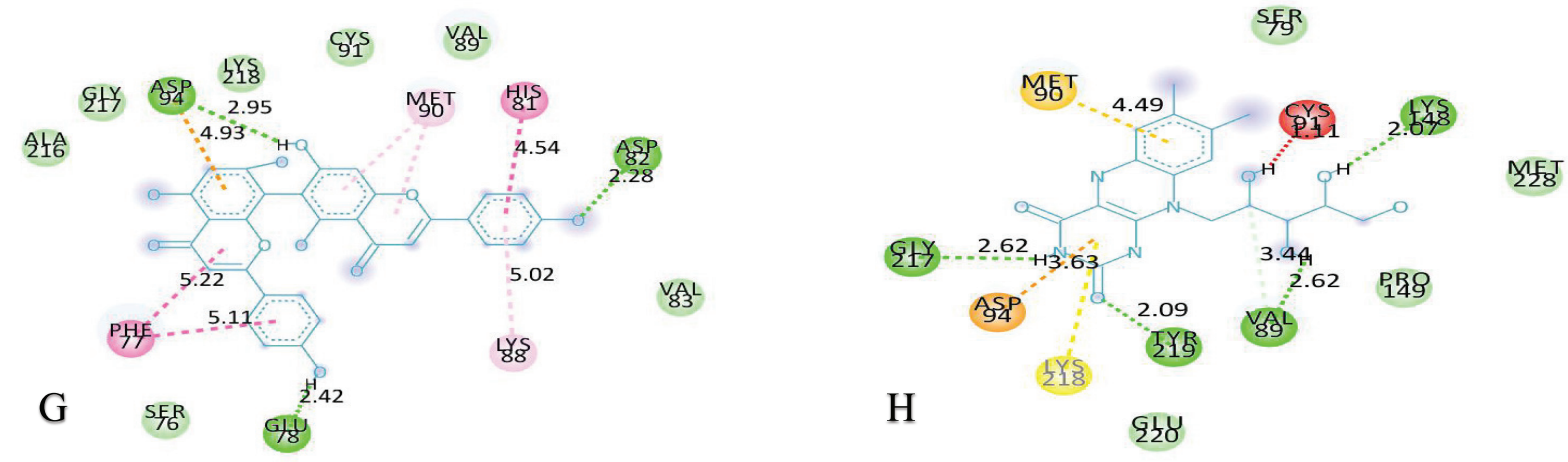

Figure 7: 2D structure of proteins interacting with ligands. A) and B) India_coat protein with Agathisflavone and Ampicillin. C) and D) Oman coat protein with ligand Agathisflavone and Albendazole. E) and F) Pakistan_coat protein with ligand Prunin 600-O-gallate and ampicillin. G) and $\mathrm{H})$ Srilanka_coat protein with ligand Agathisflavone and Ampicillin.

alpha-amylase and lipase activity [24], inhibit dioxygen or peroxide antioxidant activity reactions [25], the inhibitory activity of acetylcholinesterase and monoamine oxidase [26], enzymatic activity [27], hepatoprotective activity [28], antioxidative activity [28,29]. Flavonoids 3',4',5,6-tetrahydroxy-3,7-dimethoxyflavone, and prunin 600- O-gallate has some unknown role. Flavonoid luteolin 7-O-beta-D-glucosiduronic acid has antimalarial activity [30] and radical scavengers' activity [31]. The ChiLCV infection is intensified by the AV1 protein and involves spreading the virus from cell to cell. It interacts with different host cell machinery proteins to increase viral replication in host cells, resulting in symptom intensity.

Our research indicates the possible role of flavonoids as AV1 inhibitors. The flavonoids will be hyper-produced to grow virusresistant crops as further work is performed on the flavonoid inhibition studies. This research offered a new horizon for the scientists working on the plant defense mechanism against viruses and engineering resistance against these viruses.

\section{CONCLUSION}

For economically valuable crops such as chilli, ChiLCV causes considerable harm. It leads to a tremendous loss in crop production. Approximately, there are more than 400 species of pathogenic plant viruses that lead to considerable losses in yield and quality. In this research, four different $\mathrm{CP}$ of the same continent countries were analyzed and concluded as more conserved regions and sequence similarity. After screening different flavonoids and chemical compounds against viral CP AV1, we conclude that flavonoids act as a potent inhibitor against ChiLCV CP with more binding affinity than chemical compounds. These ligand molecules were prescreened for ADME properties to test their drug-likeness and bioavailability and showed no harmful effects while consuming economically prized crops end-products by a human. As the plant itself secretes these flavonoids, there is no harm to host plants. This study might be interesting for enhancing the inbuilt resistance in plants against ChiLCV. It has illustrated the interactions of ChiLCV CP with plant secreted compounds, such as flavonoids and their role in plant defense mechanism.

\section{AUTHOR CONTRIBUTIONS}

All authors made substantial contributions to conception and design, acquisition of data, or analysis and interpretation of data; took part in drafting the article or revising it critically for important intellectual content; agreed to submit to the current journal; gave final approval of the version to be published; and agree to be accountable for all aspects of the work. All the authors are eligible to be an author as per the international committee of medical journal editors (ICMJE) requirements/guidelines.

\section{FUNDING}

There is no funding to report.

\section{CONFLICTS OF INTEREST}

The authors report no financial or any other conflicts of interest in this work.

\section{ETHICAL APPROVALS}

This study does not involve experiments on animals or human subjects.

\section{REFERENCES}

1. Maurya PK, Srivastava A, Mangal M, Talukdar A, Mondal B, Solanki $\mathrm{V}$, et al. Genetic analysis for resistance to leaf curl disease in chilli peppers (Capsicum annuum L.) under specific situations. Indian J Genet Plant Breed 2019;79:741-8; doi:10.31742/IJGPB.79.4.13

2. Senanayake DMJB, Varma A, Mandal B. Virus-vector relationships, host range, detection and sequence comparison of chilli leaf curl virus associated with an epidemic of leaf curl disease of chilli in Jodhpur, India. J Phytopathol 2012;160:146-55; doi:10.1111/j.14390434.2011.01876.x

3. Chakraborti S, Bhowmik S, Senapati A. Assessing the impacts of safer management strategies against brinjal pests with emphasis on understorey repellent crop. J Entomol Res 2014;38:201-8.

4. Chattopadhyay B, Singh AK, Yadav T, Fauquet CM, Sarin NB, Chakraborty S. Infectivity of the cloned components of a begomovirus: DNA beta complex causing chilli leaf curl disease in India. Arch Virol 2008;153:533-9; doi:10.1007/s00705-007-0017-2

5. George B, Alam CM, Kumar RV, Gnanasekaran P, Chakraborty S. Potential linkage between compound microsatellites and 
recombination in geminiviruses: evidence from comparative analysis. Virology 2015;482:41-50; doi:10.1016/j.virol.2015.03.003

6. Gnanasekaran P, Kumar RK, Bhattacharyya D, Kumar RV, Chakraborty S. Multifaceted role of geminivirus associated betasatellite in pathogenesis. Mol Plant Pathol 2019;20:1019-33; doi:10.1111/ mpp. 12800

7. Sinha DP, Saxena S, Kumar S, Singh M. Detection of pepper leaf curl virus through PCR amplification and expression of its coat protein in Escherichia coli for antiserum production. Afr J Biotechnol 2011;10:3290-5; doi:10.5897/ajb10.1529

8. Padidam M, Beachy RN, Fauquet CM. A phage single-stranded DNA (ssDNA) binding protein complements ssDNA accumulation of a geminivirus and interferes with viral movement. J Virol 1999;73:160916; doi:10.1128/jvi.73.2.1609-1616.1999

9. Singh Y, Gupta R. Novel S-enantioselective lipase TALipB from Trichosporon asahii MSR54: heterologous expression, characterization, conformational stability and homology modeling. Enzyme Microb Technol, 2016;83: 29-39; doi:10.1016/j.enzmictec.2015.11.003

10. Gaur RK, Prajapat R, Marwal A, Sahu A, Rathore MS. First report of a begomovirus infecting Mimosa pudica in India. J Plant Pathol 2011; Vol.93 No.4, Supplement pp.S4.80 ref.2 doi:10.4454/jpp.v93i4.2394

11. Zakaryan H, Arabyan E, Oo A, Zandi K. Flavonoids: promising natural compounds against viral infections. Arch Virol 2017;162:2539-51; doi:10.1007/s00705-017-3417-y

12. Brown CR. Antioxidants in potato. Am J Potato Res 2005;82:163-72; doi:10.1007/BF02853654

13. Prabahar A, Swaminathan S, Loganathan A, Jegadeesan R. Identification of novel inhibitors for tobacco mosaic virus infection in Solanaceae plants. Adv Bioinformatics 2015;2015, Article ID 198214, 9 pages; doi:10.1155/2015/198214

14. Kartasasmita RE, Herowati R, Gusdinar T. Docking study of quercetin derivatives on inducible nitric oxide synthase and prediction of their absorption and distribution properties. J Appl Sci 2010;10:3098-104; doi:10.3923/jas.2010.3098.3104

15. Zhang S, Yang X, Sun M, Sun F, Deng S, Dong H. Riboflavin-induced priming for pathogen defense in Arabidopsis thaliana. J Integr Plant Biol 2009;51:167-74; doi:10.1111/j.1744-7909.2008.00763.x

16. Ferreyra MLF, Rius SP, Casati P. Flavonoids: biosynthesis, biological functions, and biotechnological applications. Front Plant Sci 2012;3:1-5; doi:10.3389/fpls.2012.00222

17. Kikuchi K, Matsushita N, Suzuki K, Hogetsu T. Flavonoids induce germination of basidiospores of the ectomycorrhizal fungus Suillus bovinus. Mycorrhiza 2007;17: 563-70; doi:10.1007/s00572-0070131-8

18. Yamasaki H, Sakihama Y, Ikehara N. Flavonoid-peroxidase reaction as a detoxification mechanism of plant cells against $\mathrm{H}_{2} \mathrm{O}_{2}$. Plant Physiol 1997;115:1405-12; doi:10.1104/pp.115.4.1405

19. Jansen MAK, den Noort REV, Adillah Tan MYA, Prinsen E, Lagrimini LM, Thorneley RNF. Phenol-oxidizing peroxidases contribute to the protection of plants from ultraviolet radiation stress. Plant Physiol 2001;126:1012-23; doi:10.1104/pp.126.3.1012

20. Parniske M, Ahlborn B, Werner D. Isoflavonoid-inducible resistance to the phytoalexin glyceollin in soybean rhizobia. J Bacteriol 1991;173:3432-9; doi:10.1128/jb.173.11.3432-3439.1991
21. Zubair M, Ali Zaidi SSE, Shakir S, Amin I, Mansoor S. An insight into cotton leaf curl multan betasatellite, the most important component of cotton leaf curl disease complex. Viruses 2017;9:1-2; doi:10.3390/ v9100280

22. Samanta A, Das G, Das SK. Roles of flavonoids in plants. Carbon N Y 2011;100:6.

23. Singh RP, Gu M, Agarwal R. Silibinin inhibits colorectal cancer growth by inhibiting tumor cell proliferation and angiogenesis. Cancer Res 2008;68:2043-50; doi:10.1158/0008-5472.CAN-07-6247

24. Kusano R, Ogawa S, Matsuo Y, Tanaka T, Yazaki Y, Kouno I.A-amylase and lipase inhibitory activity and structural characterization of Acacia Bark Proanthocyanidins. J Nat Prod 2011;74:119-28; doi:10.1021/ np100372t

25. Bazylko A, Stolarczyk M, Derwiska M, Kiss AK. Determination of antioxidant activity of extracts and fractions obtained from Galinsoga parviflora and Galinsoga quadriradiata, and a qualitative study of the most active fractions using TLC and HPLC methods. Nat Prod Res 2012;26:1584-93; doi:10.1080/14786419.2011.582469

26. Urbain A, Marston A, Grilo LS, Bravo J, Purev O, Purevsuren $\mathrm{B}$, et al. Xanthones from Gentianella amarella ssp. acuta with acetylcholinesterase and monoamiiu oxidase inhibitory activities. J Nat Prod 2008;71:895-7; doi:10.1021/np0706901

27. Luca VD, Ibrahim RK. Enzymatic synthesis of polymethylated flavonols in Chrysosplenium americanum. I. Partial purification and some properties of S-adenosyl-1-methionine: flavonol 3-, 6-, 7-, and 4'-O-methyltransferases. Arch Biochem Biophys 1985;238:596-605; doi:10.1016/0003-9861(85)90205-X

28. Anand KK, Gupta VN, Rangari V, Singh B, Chandan BK. Structure and hepatoprotective activity of a biflavonoid from Canarium manii. Planta Med 1992;58:493-5; doi:10.1055/s-2006-961533

29. Tang W, Hioki H, Harada K, Kubo M, Fukuyama Y. Antioxidant phenylpropanoid-substituted epicatechins from Trichilia catigua. J Nat Prod 2007;70:2010-3; doi:10.1021/np0703895

30. Ovenden SPB, Cobbe M, Kissell R, Birrell GW, Chavchich M, Edstein MD. Phenolic glycosides with antimalarial activity from Grewillea "Poorinda Queen.” J Nat Prod 2011;74:74-8; doi:10.1021/np100737q

31. Dapkevicius A, Beek TAV, Lelyveld GP, Veldhuizen AV, Groot AD, Linssen JPH, et al. Isolation and structure elucidation of radical scavengers from Thymus vulgaris leaves. J Nat Prod 2002;65:892-6; doi:10.1021/np010636j

How to cite this article: Jeyaraj G, Mohideen HS, Geetanjali AS. Ab-initio modeling and docking evaluation of geographically derived coat proteins of chilli leaf curl virus with flavonoids and chemical compounds. J Appl Biol Biotech 2021;9(S1):40-51. 